\title{
Repeat Transabdominal Laparoscopic Encerclage
}

\author{
SR Raghuwanshi, ST Shashikala, KA Rao \\ Bangalore Assisted Conception Center, Bengaluru, Karnataka, India
}

Correspondence: SR Raghuwanshi, Bangalore Assisted Conception Center, 6/7 Kumara Krupa Road, High Grounds Bengaluru-560001, Karnataka, India, e-mail: drshashiraghuwanshi@yahoo.com

\section{ABSTRACT}

We report here the first case of placement of a repeat laparoscopic abdominal cervicoisthmic cerclage by using a suture passer. The report is on a 34-year-old $G_{5} P_{1} L_{1} A_{3}$ with a history of repeated second trimester pregnancy loss with one successful laparoscopic cerclage term LSCS delivery, again underwent repeat laparoscopic abdominal encerclage in present pregnancy at 12 weeks using suture passer suprapubically. Patient underwent procedure safely with blood loss less than $40 \mathrm{ml}$ and discharged after 24 hours of observation without any postoperative complication. Repeat laparoscopic abdominal encerclage can be done safely in previous LSCS patient who had term pregnancy following laparoscopic abdominal cerclage using a simple instrument suture passer without any postoperative complication or discomfort to the patient.

Keywords: Laparoscopy, Abdominal cerclage, Suture passer.

\section{INTRODUCTION}

Cervical insufficiency (previously known as cervical incompetence) is characterized by painless cervical dilation and pregnancy loss in and beyond second trimester. By rough estimates, this condition affects $1 \%$ of all pregnancies and up to $8 \%$ of those with second- and early third-trimester pregnancy losses. ${ }^{1}$ Although there is no standardized method to detect cervical insufficiency, studies have assessed cervical length by ultrasound or physical examination and obstetrical history with limited success. ${ }^{2}$ Once the presumed diagnosis of cervical insufficiency has been made, the most commonly used method to treat this disorder is a purse string stitch around the cervix commonly by Mc Donald's or Shirodkar's method and rarely by Hefner or Wurm, abdominal or Lash cerclage.

Since its detailed description nearly four decades ago by Benson and Durfee in $1965,{ }^{3}$ many case series have confirmed the value of abdominal cervicoisthmic cerclage by laparotomy in women with cervical incompetence for whom a conventional transvaginal procedure would be inadequate or impossible. In general, the procedure has been done in pregnant women, either towards the end of the first trimester or early in the second trimester with delivery rates of viable infants consistently in the range of $85 \pm 90 \%$ (Mahran, 1978; Novy, 1991; Cammarano et al, 1995; Gibb and Salaria, 1995; Topping and Farquharson, 1995; Craig and Fliegner, 1997; Turnquest et al, 1999).

The traditional approach for an abdominal cerclage via an open laparotomy incision has the disadvantage that it requires

Date of Received: 03-01-11

Date of Acceptance: 26-04-11

Date of Publication: May 2011 at least two laparotomies with significant morbidity and hospital stays. The laparoscopic approach should be the preferred option in patients with cervical insufficiency requiring abdominal cerclage placement. ${ }^{4}$ Here, we describe the unique case of repeat transadmominal cerclage by laparoscopy in a pregnant patient who already delivered a baby by cesarean section after laparoscopic abdominal cervicoisthmic cerclage in previous pregnancy.

\section{CASE REPORT}

A 34-year-old, 12 weeks pregnant lady with natural conception was electively posted for laparoscopic abdominal cervicoisthmic encerclage. She was $\mathrm{G}_{5} \mathrm{P}_{1} \mathrm{~L}_{1} \mathrm{~A}_{3}$. After 7 years of marriage, patient conceived through IVF, which was a single intrauterinre pregnancy. At around 6 weeks 3 days, she developed urinary treat infection (UTI) which ultimately ended with missed abortion for which dilatation and curettage were done. In about one year, she underwent hysteroscopy with septal resection for incomplete septum before second in vitro fertilization (IVF). She underwent IVF with four embryo transfer which resulted in quadripulate pregnancy. During follow-up she lost one fetus with other three viable fetuses of around 6 weeks, later on, fetal reduction was done and triplets were reduced to twins. At 17 weeks of gestation, patient presented with inevitable abortion for which again dilatation and curettage were done.

Unfortunately, her third IVF also ended with abortion and $\mathrm{D}$ and $\mathrm{C}$ before planning her cervical encerclage.

One year after third IVF in 2008, patient conceived naturally and this time laparoscopic abdominal cervical encerclage was done at 10 weeks 5 days of gestation with cervical length of $2.6 \mathrm{~cm}$. Elective lower segment cesarian section (LSCS) was 
done at 37 weeks 3 days during which Mersilene tape was found to be infected and hence removed. She delivered a $3.1 \mathrm{~kg}$ healthy female baby.

Again, 2 years later patient conceived naturally and in view of previous history of repeated abortion and successful laparoscopic abdominal cervical encerclage, she was posted for laparoscopic abdominal encerclage which was done at 12 weeks of gestation.

\section{BRIEF DESCRIPTION OF SURGICAL TECHNIQUE}

Under general anesthesia, with the patient in the dorsal lithotomy position, Foley's catheterization was done. Pneumoperitoneum with Verres needle created. One supraumbilical $10 \mathrm{~mm}$, two $5 \mathrm{~mm}$ lateral and one suprapubic ports introduced. After opening uterovesicle (UV) fold (Fig. 1) bladder separation was done which was little difficult because of the previous LSCS and the bladder was pushed down (Fig. 2). Suture passer was introduced suprapubically (Fig. 3) and advanced through the substance of cervix medial to uterine artery in anterior to posterior direction on one side (Fig. 4). Mersilene tape of $5 \mathrm{~mm}$ breadth and $30 \mathrm{~cm}$ length is first prepared by cutting the needles from either end. One end of the tape is grasped with suture passer (Koebler's needle) (Fig. 4) and pulled through cervix from posterior to anterior of cervix (Fig. 5). Cervix is made prominent when required with the help of sponge on ring forceps vaginally. Same procedure is repeated on the other side (Fig. 6). After verifying that the tape is lying flat and smoothly around the posterior surface of uterus (Fig. 7), knot was tied anteriorly (Fig. 8) and extra portion of tape cut out. Total blood loss was less than around $40 \mathrm{ml}$. Position of the tape confirmed by transvagianl sonography which was at the level of isthmus well below uterine cavity and amniotic sac. Pelvis was lavaged with normal saline, homeostasis confirmed, and the laparoscopy was terminated in usual manner. At the end of procedure, bladder integrity confirmed cystoscopically. Postoperative course of the patient was uneventful. Patient was kept under observation for 24 hours and discharged the very next day.

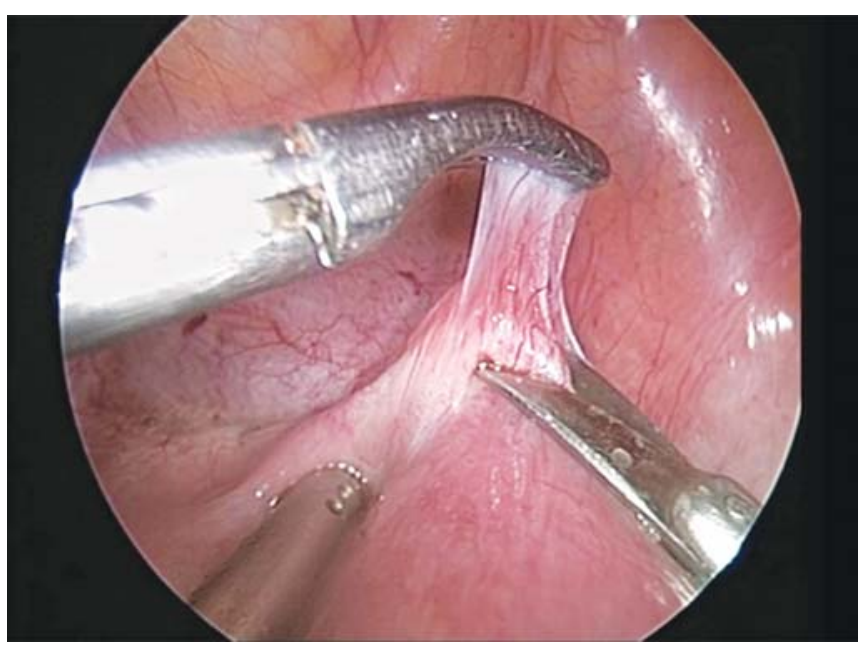

Fig. 1: Opening uterovesicle fold
Our institutional board at Bangalore Assisted Conception Center, Bengaluru, India, does not require approval for this case report because it describes standard surgical procedure.

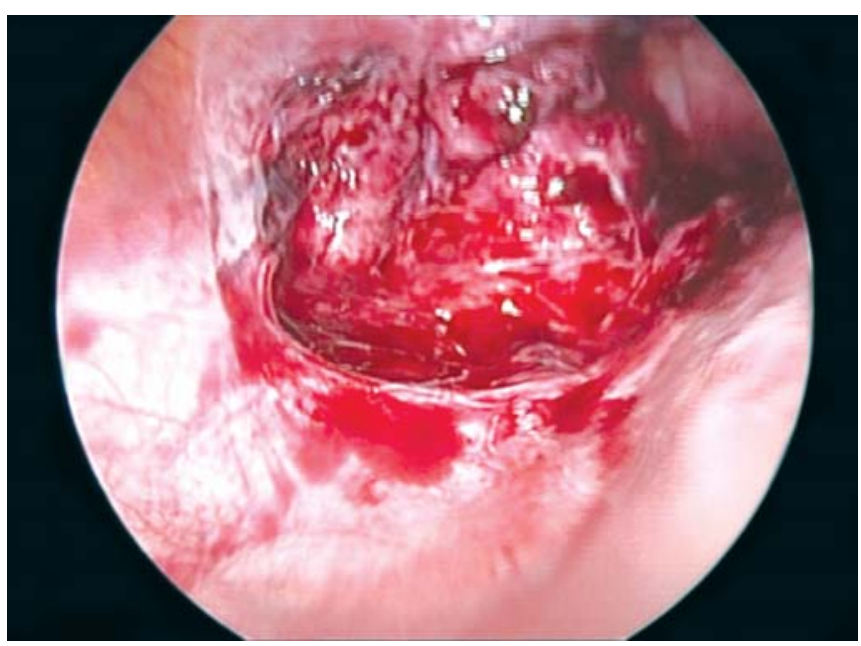

Fig. 2: Bladder pushed down

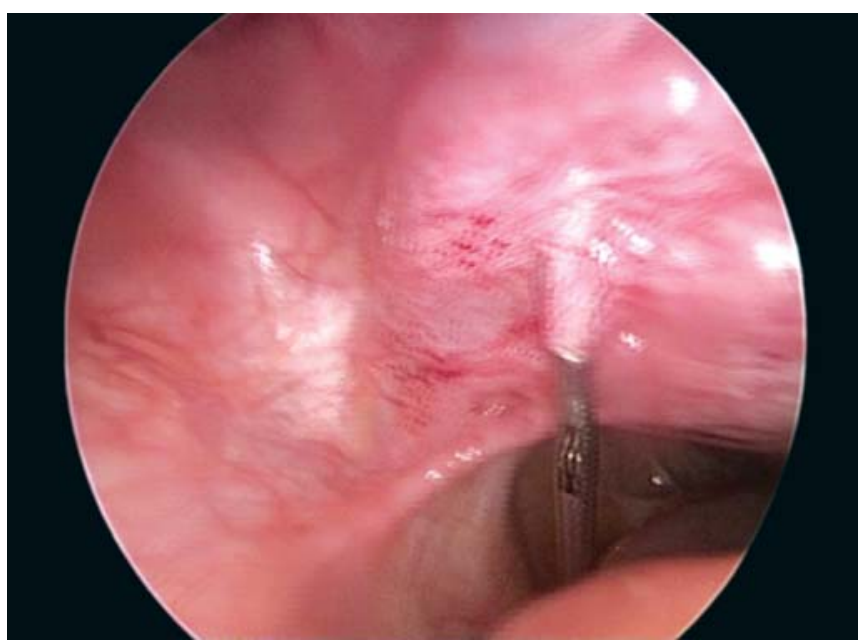

Fig. 3: Suture passer needle in peritoneal cavity anterior to uterus

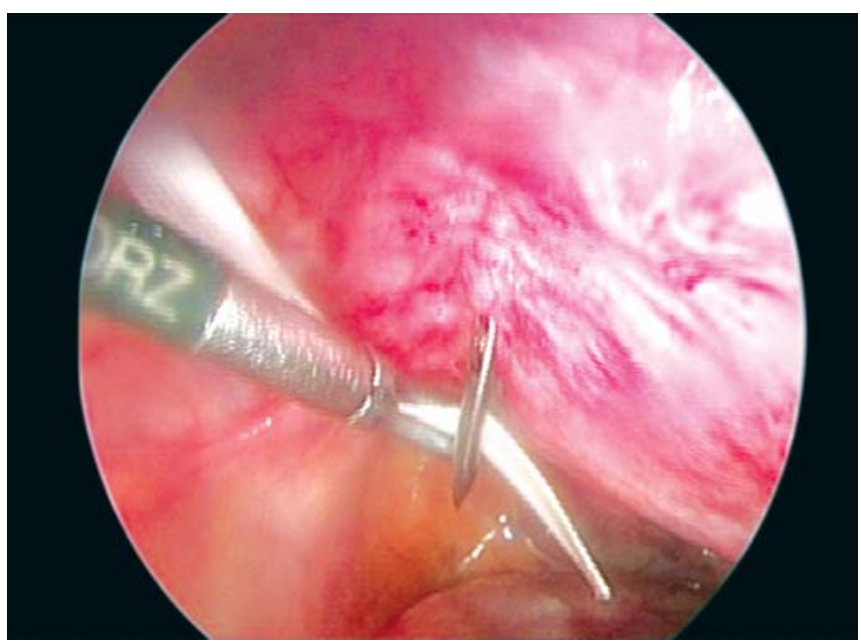

Fig. 4: Suture passer needle posteriorly through the substance of cervix grabbing one end of Mersilene tape 


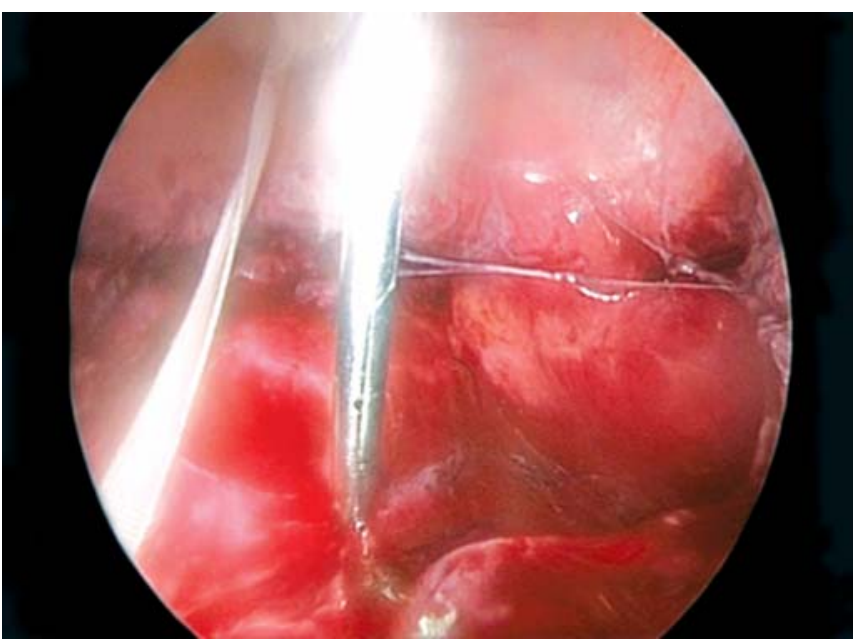

Fig. 5: Pulled end of the Mersilene tape anterior to cervix and re-entry of needle for other end

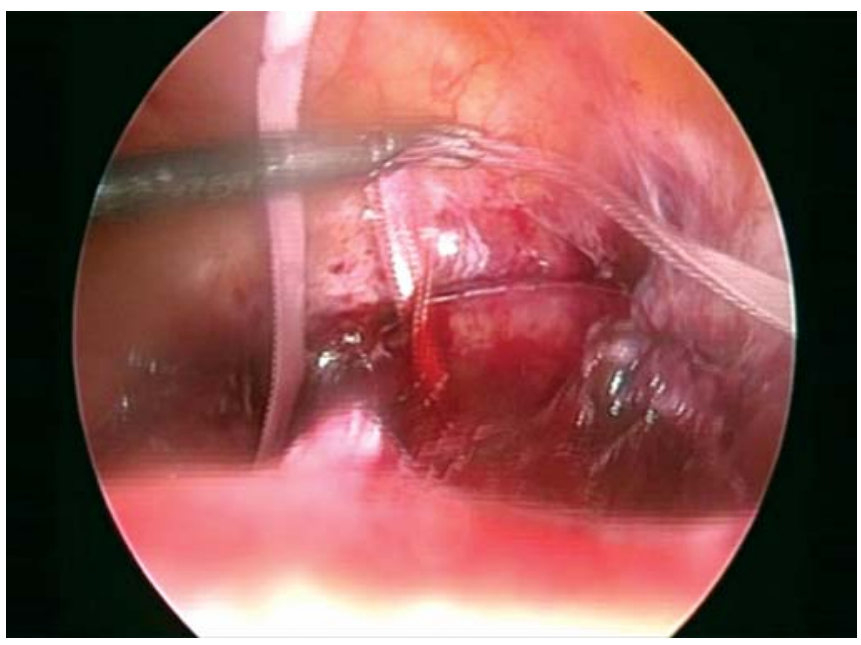

Fig. 6: Pulled both ends of Mersilene tape anterior to cervix

\section{DISCUSSION}

Cervical incompetence occurs in 0.5 to $1 \%$ of all pregnancies, has a recurrence risk of $\sim 30 \%$ and typically presents in the second trimester as pelvic pressure and cervical dilation in the absence of uterine activity or ruptured membranes. ${ }^{5}$ The etiology of cervical incompetence can be classified $\mathrm{as}^{5}$ a mechanical failure of the cervix to remain closed against the increasing uterine distention and pressure or a functional failure due to premature cervical ripening. ${ }^{5}$ The cervical cerclage to reinforce the cervical integrity is conventionally done through vaginal approach.

In 1965, Benson and Durfee ${ }^{3}$ introduced an alternative to the vaginal approach - the placement of a cerclage at the cervical isthmus; a noose-like suture positioned around the isthmus in the avascular space above the cardinal and uterosacral ligaments placed by laparotomy. ${ }^{6}$ This technique was intended when the vaginal approach was not feasible due to altered cervical anatomy, the indication was extended by Novy ${ }^{7}$ to include a failed transvaginal cerclage in a previous pregnancy. Cervicoisthmic cerclage reviews quote a successful pregnancy outcome rate from 76.5 to $100 \%$, however, the morbidity

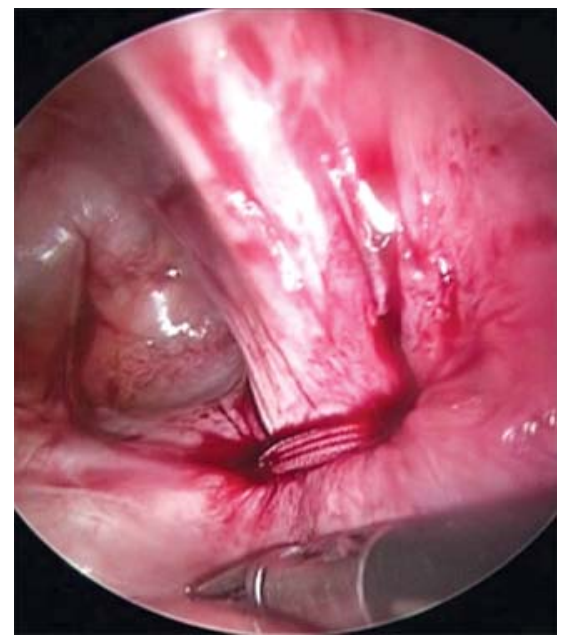

Fig. 7: Postsurface of uterus with smooth horizontal tape at isthmus

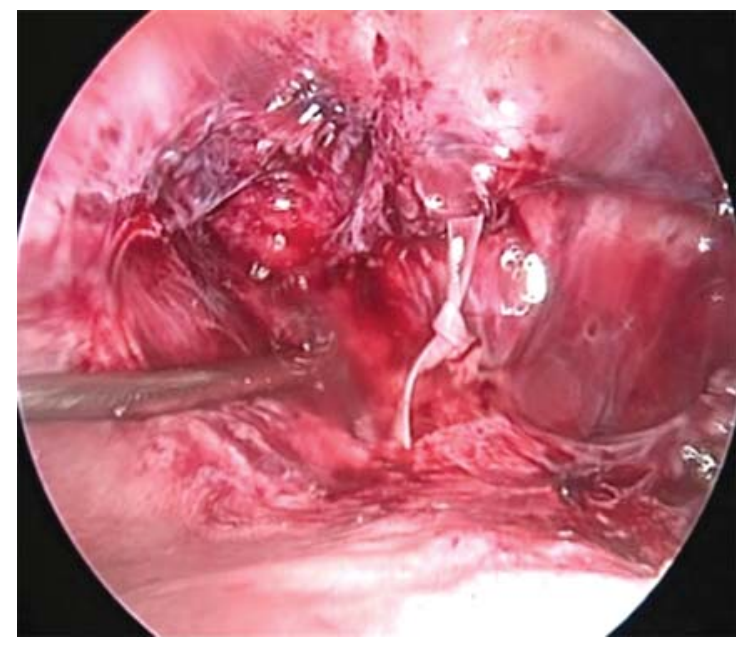

Fig. 8: Knot tied anteriorly

associated with the surgical procedure is significant (0-25\%). ${ }^{8}$ In an era when endoscopic surgery provides a minimally invasive alternative with documented benefit over the traditional laparotomy approach, it has been proposed that the cervicoisthmic cerclage could be contemplated laparoscopically. ${ }^{9}$

Here, we have reported a case of repeat transabdominal cerclage in a patient who successfully delivered a term healthy baby in previous pregnancy after laparoscopic abdominal cerclage and whose cerclage has been removed during cesarean section because of infection. Second pregnancy with cerclage in situ, which was placed in first pregnancy in patients, has been described in literature ${ }^{10}$ but in our case old tape is removed in previous delivery and laparoscopic abdominal cerclage is redone in present pregnancy.

One would expect difficulties in doing cerclage in a patient of previous LSCS because of adhesions at uterovesicle fold and fibrosis. In these cases cerclage done by Mersilene tape with needle holder can be difficult as the force required is more and that force is applied from a long distance, i.e. length of needle holder, that increase the chances of injury to bladder, bowel and cervix. This cerclage is done by an innovative simple 
procedure with the help of port closure needle suture passer (Fig. 9), which is a short instrument and the force is applied without any angulation. It is introduced suprapubically with very good control while advancing through the substance of cervix thus can avoid inadvertent bowel injury posteriorly and as the direction of introduction is anterior to posterior thus avoids bladder and ureteric injury as well.

In our case both the times cerclage has been done laparoscopically which obviously has very less morbidity than laparotomy, otherwise patient has to undergo in total four laparotomies which could largely have increased the morbidity of the patient. And, as the suture is passing through the substance of uterus, there is no external compression of uterines, so no chance of placental blood flow compromise which can happen by putting the cerclage through window in broad ligament.

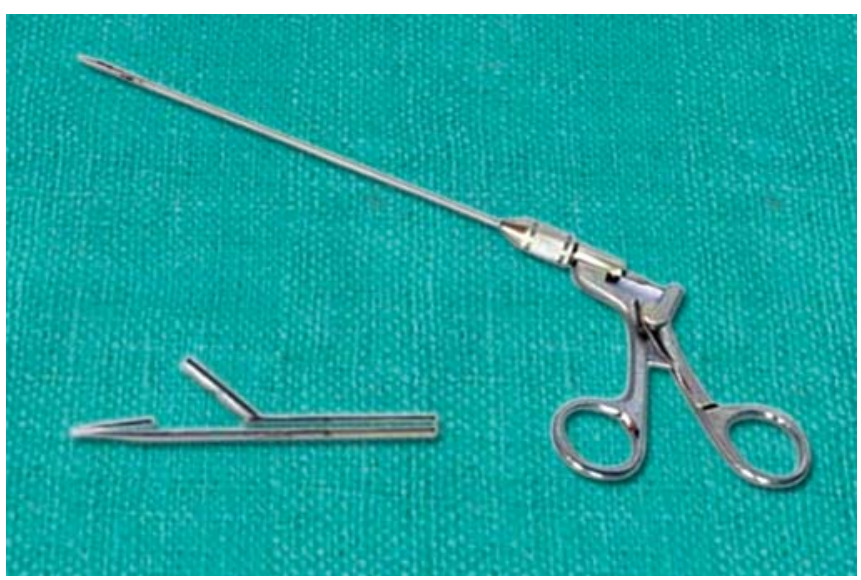

Fig. 9: Suture passer

\section{COMMENT}

This simpler method we described above has advantage of safety, ease and control making the laparoscopic cerclage placement more easy and safe even for the second time in a patient of previous cerlage and cesarian section.

\section{REFERENCES}

1. Drakeley AJ, Roberts D, Alfirevic Z. Cervical stitch (cerclage) for preventing pregnancy loss in women. Cochrane Database Syst Rev 2003;1:CD003253.

2. Harger J. Cerclage and cervical insufficiency: An evidence-based analysis. Obstet Gynecol 2002;100:1313-27.

3. Benson RC, Durfee RB. Transabdominal cervicouterine cerclage during pregnancy for treatment of cervical incompetency. Obstet Gynecol 1965;25:145-55.

4. Suzuki Y, Little SE, Smith NA, Oxford CM, McElrath TF. Einarsson JI Journal of Minimally Invasive Gynecology 2009;16:S1eS51.

5. Shennan A, Jones B. The cervix and prematurity: Aetiology, prediction and prevention. Semin Fetal Neonatal Med 2004;9:471-79.

6. McDonald IA. Suture of the cervix for inevitable miscarriage. J Obstet Gynaecol Br Emp 1957;64:346-50.

7. Novy MJ. Transabdominal cervicoisthmic cerclage for the management of repetitive abortion and premature delivery. Am J Obstet Gynecol 1982;1:44-54.

8. Lotgering FK, Gaugler-Senden IP, Lotgering SF, Wallenburg HC. Outcome after transabdominal cervicoisthmic cerclage. Obstet Gynecol 2006;107:779-84.

9. Scibetta JJ, Sanko SR, Phipps WR. Laparoscopic transabdominal cervicoisthmic cerclage. Fertil Steril 1998;69:161-63.

10. Drakeley AJ, Quenby S, Farquharson RG. Mid-trimester loss-appraisal of a screening protocol. Hum Reprod 1998;13:1975-80. 